\title{
PREVENTION IS BEST ESCAPE TO COVID-19
}

\section{U. C. SRIVASTAVA ${ }^{1}$ and SIPPY SINGH ${ }^{2}$}

${ }^{I}$ Department of Zoology, University of Allahabad and Treasurer-The National Academy of Sciences, India Prayagraj (U.P.) ${ }^{2}$ Department of Zoology, S.S. Khanna Girls' Degree College, Prayagraj (U.P.)

\begin{abstract}
COVID-19 is an infectious outbreak which has been declared as pandemic diseases by World Health Organization. This disease caused by SARS-CoV-2 virus transmits through droplet infection. Recent research highlights the airborne transmission of this virus. Since at present there is no vaccination against COVID-19, therefore the only escape route or prevention is to follow the preventive measures suggested by government and boost one's immune system. Natural herbs, yoga, meditation and mild exercise are known to act as immunity boosters.
\end{abstract}

Key words : COVID-19, SARS-CoV-2, Immune system.

Corona Virus Disease 2019 (COVID-19) is an outbreak of pneumonia which was first reported in Wuhan, Hubei Province of Central China in December,2019. This infectious disease is caused by novel corona virus : SARS CoV-2 (Severe acute respiratory syndrome corona virus 2) which belongs to large family of single-stranded RNA viruses. The infection has been proposed to undergo zoonotic transmission spreading from animals to humans and causing respiratory, gastrointestinal, hepatic and neurological diseases (Weiss and Leibowitz,2011). This disease has spread globally covering across 72 countries till date and has been declared as pandemic by World Health Organization (WHO).WHO has declared COVID-19 as Public Health Emergency of International Concern (PHEIC).

SARS-CoV-2 virus belongs to the B lineage of the $\beta$ $\mathrm{CoVs}$ and is $7^{\text {th }}$ member with over $70 \%$ similarity in genetic sequence to SARS-nCoV (Cheng and Shan,2019). This virus has single stranded RNA as genetic material encased in a lipid layer with protein spikes embedded into the layer giving it appearance of crown, hence named corona (Fig.1).

This virus has been proposed to likely originate from bats which infects humans through other intermediate animal hosts, e.g. the civet (Paguma larvata) for SARS-CoV 2. The transmission cycle includes traversing of virus from bats to intermediate host having Ace-2 Receptor (receptor to which SARS-CoV2 binds) to humans (possessing Ace-2 receptors). Further transmission of virus among humans is through droplet infection or direct contact.

General symptoms of disease includes headache, dry cough, soar throat, runny nose, diarrhoea, conjunctivitis, headache, loss of taste or smell, skin rashes or discoloration of fingers and toes whereas serious symptoms of disease include: difficulty in breathing, shortness of breath, chest pain or pressure and loss of speech or movement. There are some basic rules or steps which can help us in preventing the infection and spread of novel corona virus such as : covering mouth and nose with mask, frequent washing of hands with soap and water or sanitization of hands with alcohol based sanitizer, maintain social distancing, avoid traveling etc. These preventive measures are best way to cope with current situation as there is no vaccine for this virus till date, so 'Prevention is better than cure' in this case.

The other most important shield against this infection could be one's immune system. Immune system of an organism helps to protect an organism from the foreign pathogens (viz., bacteria, viruses, fungus, germs, parasites) or particles (carcinogens, pollutants, toxins). It helps in recognition of self and destruction of foreign particle. When a new pathogen is introduced to an organism, it lacks immunity so it is widely spread. Immunity can be either innate (by birth) or acquired/ adaptive (after encountering with pathogen) (Fig.2). Encountering a new pathogen can induce acquired immunity. Immune health is affected by a number of factors including diet, exercise, sleep or environmental factors.

It is interesting to note that traditionally herbs are used as ayurvedic medicines which boost the immune system. It protects against stress and helps to improve health by increasing vigor, vitality and longevity. Strong immunity is the product of good digestion and it can be reset by fasting.

Some of the common natural herbs which are used to enhance and maintain immune system are :

Allium sativum (Garlic) : This perennial flowering plant belonging to family Amaryllidaceae contains rich amount of vitamin B6, thiamin, pantothenic acid, vitamin C, and minerals such as-Mn, P, Ca and Zn. Garlic also contains antioxidants that protects body against oxidative damage. Ajoene found in garlic has promising anti-leukaemic activity. Addition of garlic in diet improves cholesterol levels and lowers the risk of heart disease. At high doses, the sulfur compounds in garlic have been shown to protect against organ damage from heavy metal toxicity. It has been found to be effective against arthritis, snake and insect bites, parasites, chronic cough, and as antibiotic for infectious diseases.

Withania somnifera (Ashwagandha) : An Indian ginseng and perennial herb which belongs to family Solanaceae. It is rich source of phytochemicals like alkaloids, lactones, ste- 
roids, flavonoids and nitrogen containing compound. W.somnifera possess anti-cancer, anti-inflammatory, antioxidants, anti-diabetic, anti-stress and cardio-protective activity. It is also being used in treatment of Parkinson's and Alzheimer's disease .

Camellia sinensis : Green tea has been widely used in traditional Indian and Chinese medicine as it is rich in antioxidants, polyphenols and flavonoids which helps in reducing stress (due to caffeine content) and fatigue. It has antiinflammation property that helps in arthritis. Green tea is very popularly used in managing weight loss, Type 2 diabetes and it also reduces chances of mortality due to cardiovascular and cancer problem.

Foeniculum vulgare (Fennel) : Perennial herb belonging to Apiaceae family contains minerals ( $\mathrm{Ca}, \mathrm{K}, \mathrm{Mg}, \mathrm{P}, \mathrm{Na}, \mathrm{Fe}$ and $\mathrm{Zn}$ ), vitamins (thiamine, riboflavin, niacin, vitamin $\mathrm{C}$, vitamin A, vitamin $\mathrm{K}$ ), lipids and proteins. It has been reported that the essential oil of mature fruit exhibits antiviral properties (Orhan et al.,2012). Fruits are rich in phenolic compounds which have antioxidant properties and reduces oxidative stress. It possesses antimicrobial, anti-inflammatory, antiallergic, anticolitic, antitumor, cytotoxic, antidiabetic and diuretic activity (Badgujar et al.,2014) and also helps in preventing cardiovascular diseases, cancer and inflammation.

Nigella sativa (Black Cumin) : Belongs to family Rananculaceae. All the parts of this herb i.e. roots, shoots and seeds show antioxidant, anti-inflammatory, anticancer and antibacterial property. It contains alpha-hederin, a water soluble pentacyclic triterpene and saponin, a potential anticancer agent. Seeds are rich source of active compound thymoquinone, thymohydroquinone and dithymoquinone, alkaloids nigellicimine and nigellicimine. It exhibits neuroprotective effect due to antioxidant, free radical scavenging, and anti-inflammatory properties. N.sativa oil shows nephroprotective effect which helps in reducing serum urea and creatinine levels. It possesses cardiovascular protective and anti-asthmatic activity and also prevents formation of gastric ulcer (Ahmad et al.,2013).

Zingiber officinale (Ginger) : This herbaceous perennial belongs to Zingiberaceae family. Its rhizome, ginger root are widely used as spices and folk medicines. It contains $79 \%$ water, $18 \%$ carbohydrates, $2 \%$ protein, and $1 \%$ fat with vitamin $\mathrm{B} 6$ and the dietary minerals- $\mathrm{Mg}$ and $\mathrm{Mn}$. Rich source of antioxidants and has antibacterial, antiviral and antiinflammation properties. Used for prevention of nausea, vomiting and common flu. It is also used for menstrual cramps, osteoarthritis, diabetes, migraine headaches and as antacid.

Ocimum sanctum (Tulsi) : Aromatic perennial herb belonging to family Lamiaceae and commonly known as holy basil or tulsi. Eugenol is the major phenolic compound present in tulsi and also includes gallic acid, vanillin, caffeic acid, chlorogenic acid etc. Antioxidant ursolic acid provides protection against lipid peroxidation in liver and heart. Oil and linolenic acid present in O.sanctum shows anti-inflammatory activity prevents gastric ulcer. Extracts are used for eye drop preparations for treatment of glucoma, cataract, chronic conjunctivitis and other painful eye diseases. Fresh leaves are used to treat chronic fever, dysentery, hemorrhage, dyspepsia, cold, checks vomiting, malaria, diabetes mellitus, skin problem and has anthelmintic activity. It possesses good antistress, analgesic, anti-hyperlipidemic, antioxidant potentials and anti-carcinogenic property.

Curcuma longa (Turmeric) : Belongs to family Zingiberaceae. Roots are used as spice. Root has volatile oil, contains turmerone and curcuminoids as coloring agents. It also has Curcumin \& variety of sesquiterpene. It has potent anti-inflammatory, antioxidant, antimicrobial and carminative property and helps to improve symptoms of depression, asthma and arthritis. It also helps in colon cancer treatment.

Linum usitatissimum (Linseed, flax seeds) : Used for protection against Cancer, lowering cholesterol and improving heart health, blood sugar. Helps in constipation and protects against radiation.

Apart from the above mentioned herbs many others are also used because of their potent activity and immune enhancing properties eg. Astragalus (Mongolian milkvetch), Silybum marianum (Milk thistle), Glycyrrhiza glabra (Liquorice) etc. Nutritional values of dry fruits, cereals, milk \& milk products, Jaggery, vegetables etc. cannot be ignored as they also act as immunity boosters because they are rich sources of vitamins and minerals. Vitamin C (Ascorbic acid) are wonderful antioxidants which help in neutralizing free radicals and thereby protect body against oxidative damage, it also plays important role in cure of common cold, protection against cancer, heart diseases and other physiological disorders. Similarly Vitamin E and beta carotene also helps in maintaining proper functioning of body and healthy immune system.

Regular intake of healthy diet along with proper physical activities can gear the rate of immune response. Intake of healthy food, tension free life, 6-8 hrs of sleep, Yoga/ Meditation, avoiding alcohol, avoiding cigarette, control in heart problem, diabetes and asthma etc. are some of tips which can act as immunity boosters.

It is truly said that 'Prevention is better than cure' and for present case it is the best option left out. So we need to follow these important and simple steps:

\section{Avoid handshakes}

2. Avoid going to crowded places

3. Avoid public transport and unnecessary traveling.

4. Maintain social distancing.

5. Wash your hand thoroughly with soap for at least $20 \mathrm{sec}$.

6. Sanitize your hand with alcohol based sanitizer.

7. Break the chain of infection by remaining in isolation if you get infected. 
8. Clean your home regularly.

9. After returning to home from outside wash your clothes.

10. Avoid smoking and drinking alcohol.

11. Cover mouth and nose with masks.

12. Take antioxidant rich foods to increase your immunity.

13. Never eat uncooked meat.

14. Exercise/yoga/meditation regularly.

15. Fasting : $12-16 \mathrm{hrs}$.

16. Have good sleep and avoid taking tension.

As per the recent study, the novel corona virus has been reported to follow airborne transmission and the virus can remain in air for nearly 8 hours, which is really matter of prime concern (Morawska and Mitton,2020). Therefore, everyone needs to be extra cautious and follow the above mentioned measures.

The present scenario of COVID-19 situation is really alarming as there is no vaccine developed till date and this leaves us with two options : either to let the virus infect us or prepare oneself against the attack of virus and shield our body. The latter can be achieved either by following the preventive measures proposed by the government or by mounting our immune system which can fight against the virus and neutralize it's effect. So the knowledge of the above mentioned immunity boosters along with regular exercise, Yoga/meditation can be very beneficial for all of us in current situation.

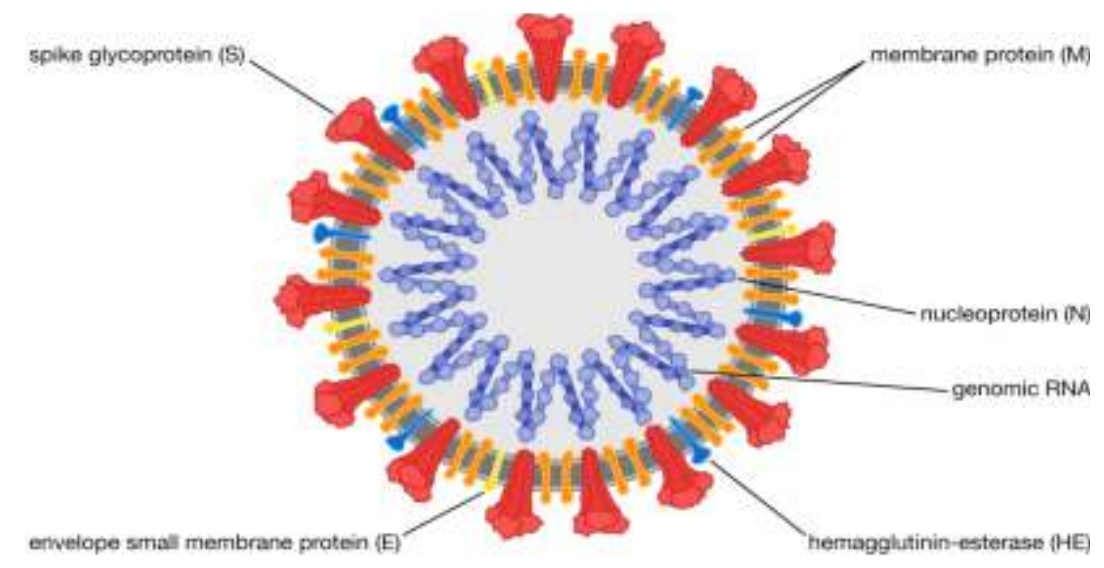

D Encydopedia Britannica inc.

Fig. 1 Structure of SARS-CoV-2

Source : Encyclopedia Britannica, Inc./Patrick O'Neil Riley.

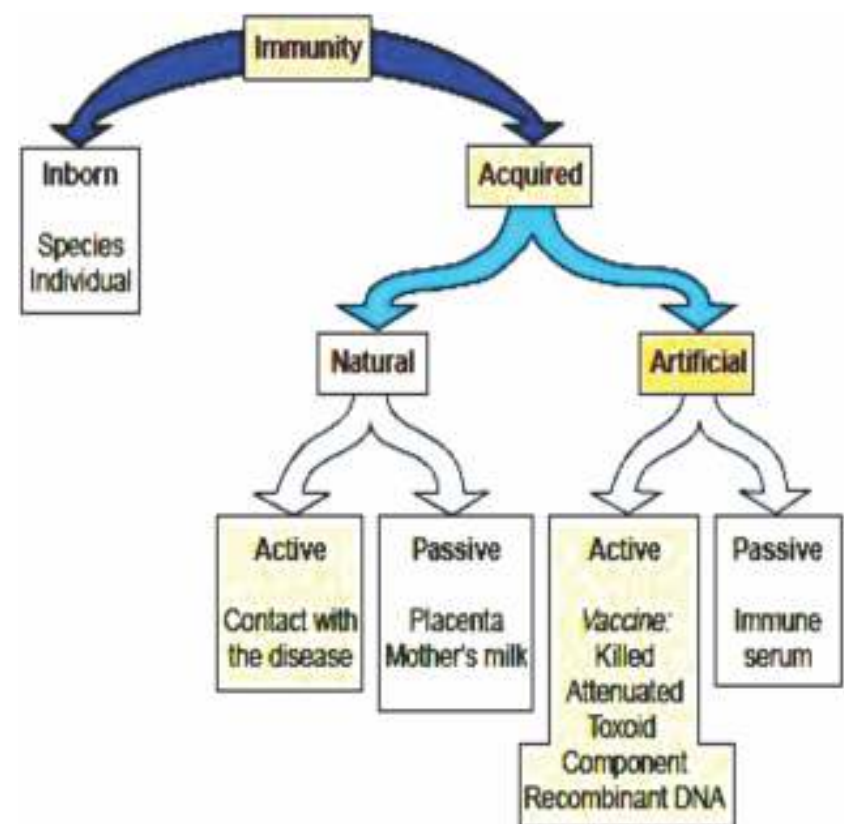

Fig. 2 Types of Immunity

Sources : http://encyclopedia.lubopitko-bg.com/Immunity.html 


\section{(3) coronavirus (-)}

\section{PREVENTION}

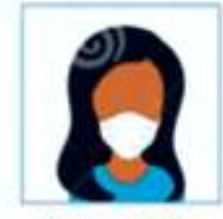

Wear a mask

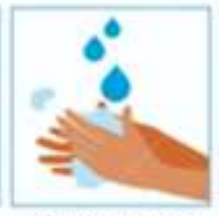

Washing hands

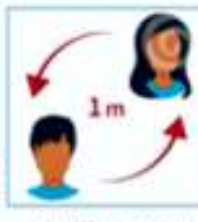

Avoid contoct

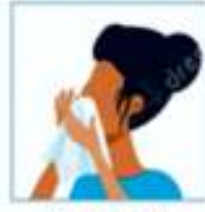

Cover your sneete and cough

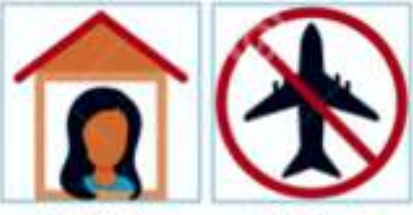

stay home

Not trovel

SYMPTOMS

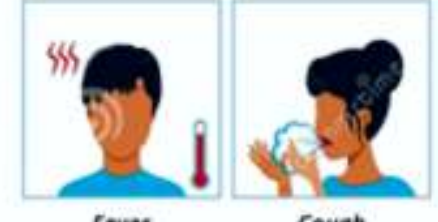

Fever

Cough

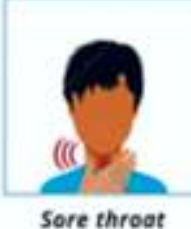

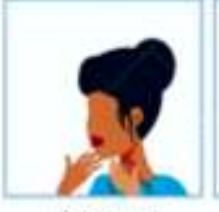

Dyspnea

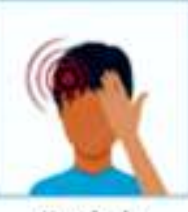

Headoche

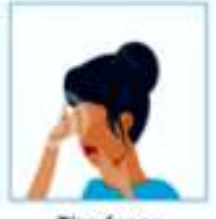

Tiredness

(ด) drenmstime.com

10 176764264 CLeontermaboce

\section{REFERENCES}

Ahmad, A.; Husain, A.; Mujeeb, M.; Khan, S. A.; Najmi, A. K.; Siddique, N. A. and Anwar, F. (2013). A review on therapeutic potential of Nigella sativa : A miracle herb. Asian Pacific Journal of Tropical Biomedicine, 3(5) : 337-352.

Badgujar, S. B.; Patel, V. V. and Bandivdekar, A. H. (2014). Foeniculum vulgare Mill : A review of its botany, phytochemistry, pharmacology, contemporary application and toxicology. BioMed. Research International.

Cheng, Z. J. and Shan, J. (2019). Novel coronavirus : Where we are and what we know. Infection 2019; Epub. ahead of print.

Morawska L. and Mitton, D. K. (2020). It is time to address airborne transmission of COVID-19. Clinical Infectious Diseases, Ciaa 939, https://doi.org/10.1093/cid/ciaa939.

Orhan, I. E.; Ozcelik, B.; Kartal. M. and Kan, Y. (2012). Antimicrobial and antiviral effects of essential oils from selected Umbelliferae and Labiatae plants and individual essential oil components. Turkish Journal of Biology, 36(3) : 239-246.

Weiss, S. R. and Leibowitz, J. L. (2011). Coronavirus pathogenesis. Adv. Virus Res 2011; 81 : 85-164. CCTV.COM. A 30-hour old infant in Wuhan diagnosed and mother-to-child infection suspected. Available at: http://m.news.cctv.com/2020/02/05/ARTIywVxQICUUURSIWzSzvkf 200205.shtml. 